\title{
artículos
}

\section{Clasificaciones y Definiciones: notas para un bibliotecario ${ }^{1}$}

\author{
Pablo González Casanova \\ Director del Centro de Investigaciones \\ Interdisciplinarias en Humanidades de \\ la UNAM. Torre II de Humanidades, \\ piso 4, Ciudad Universitaria, México \\ D.F. tel: 6230026 \\ E-mail: casanova@ servidor.unam.mx
}

\section{RESUMEN}

$\boldsymbol{T}$ oda clasificación es histórica y relativa, sujeta a la práctica de quienes la emplean, las variaciones no se deben sólo a los intereses prácticos de quienes clasifican y definen, también están presentes las variaciones reales de los conjuntos y subconjuntos de los universos y partes que se mueven; también están presentes elementos ideológicos, que ocultan, prohíben, que obligan a clasificar de una manera determinada. Elementos que también inciden en el desarrollo de los acervos. La historia de las clasificaciones muestra que éstas sirven para ordenar, establecer diferencias, jerarquías, géneros.

Actualmente, la interdisciplina tiene profundas implicaciones y exige una nueva organización de conocimiento en las bibliotecas, en donde los libros pueden ser objeto de clasificaciones múltiples, bajo órdenes que sean racionales, funcionales, útiles con capacidad de integrar nuevas ramas del conocimiento; estas clasificaciones deberán considerar los límites, rupturas o discontinuidades. Además considerar que un mismo libro se puede clasificar dentro de dos o más temas. En la clasificación de los libros se deben considerar factores tales como: facilitar su localización rápida y oportuna, hacer posible la relación entre libros de diversos campos del conocimiento.

\section{ABSTRACT}

$\boldsymbol{E}_{\text {ach classification is historical, relative and subject to the practice of the person using it. The variations are not }}$ solely due to the practical interests of the person who is classifying or defining. Real variations of the groupings and subgroupings, of the moving universes and its parts, are also present; as well as, ideological elements that hide, forbid, that force a classification in a certain way; elements that influence the collection development.

The history of classifications shows that they are used to order, establish diferences, hierarchies, genders.

Currently, the interdiscipline has deep implications and demands a new knowledge organization within libraries, where books may be the object of multiple classifications, following rational or functional orders, useful to integrate new branches of knowledge. These classifications should consider limits, ruptures or discontinuities, besides taking into consideration that a book can be classified within two or more subjects. Factors such as quick and opportune location should also be considered in book classification, for the purpose of relating books to different fields of knowledge.

$\mathrm{D}$ e la biblioteca, Borges habla en dos dimensiones. Hay una en que habla de Algo cuyo nombre es distinto. Unos lo llaman Universo, otros, Biblioteca. El Universo es una Biblioteca y sus habitantes son bibliotecarios.

La biblioteca es una esfera con una circunferencia inaccesible. Es infinita, es eterna.
Algunos bibliotecarios son pensadores e investigadores que hacen teorías de la biblioteca o que descubren las leyes de la biblioteca. (Uno de ellos llega a la conclusión cartesiana: "Tengo una biblioteca, luego existo.") Los hay que son viajeros: al cabo de siglos comprenden que "el Orden" es el desorden de la biblioteca. ${ }^{2}$
En otra dimensión, el bibliotecario Borges, llega a su lugar de trabajo: "Los rumores de la plaza - escribe-quedan atrás y entro en la Biblioteca. De una manera casi física siento la gravitación de los libros, el ámbito sereno de un orden, el tiempo disecado y conservado mágicamente[...]"

1 Este texto corresponde la la Conferencia Magistral que el Dr. Gonzáles Casanova dictó en el XIV Coloquio de Investigación Bibliotecológica.

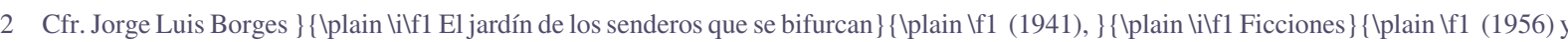
\}$\{$ \plain \ilf1 El hacedor\} \{\plain \f1 (1972). 
La idea de la clasificación y la definición pertenecen a ese orden sereno, conservado, disecado y mágico. Ese orden y sus códigos nos permiten guardar los libros y nos proporcionan la magia de encontrar aquellos que queremos, o los que quieren nuestros lectores.

Conocer ese orden es tarea técnica. Implica todo tipo de conocimientos, algunos ligados a nuestra infancia, otros a la filosofía, y a la organización del saber a lo largo de la historia con conceptos y categorías que cambian; que tienen vida, entre sentidos encontrados, y pérdidas completas de sentido tras las que buscamos descubrir qué significan nuestras definiciones y clasificaciones, nuestro instinto de orden en el desorden.

\section{EL NIÑO HACE CLASIFICACIO- NES Y DEFINICIONES}

A partir del momento en que el niño establece relaciones entre sus fines y sus medios desarrolla su inteligencia. A partir de aquel momento en que desplaza objetos distingue a los objetos por su distancia, por su peso. Más tarde se distingue a sí mismo de los objetos. El niño hace clasificaciones preverbales que al crecer expresa en palabras y refleja o construye en conceptos. Entre los 5 y los 6 años ya distingue entre el individuo y la clase o grupos o categorías a los que aquél pertenece. Pasa de las colecciones de figuras parecidas y distintas, a conjuntos de objetos que tienen una misma función, como las mesas, y a conjuntos de objetos, como los que se ponen encima de las mesas.

Así, en la niñez empieza un largo proceso en que la clasificación se enriquece con el desarrollo de muchos elementos más de la inteligencia y el saber. El niño, el hombre se vuelve cada vez más inteligente y sabe cada vez más; la niña, la mujer.

Los estudios de Piaget ${ }^{3}$ sobre epistemología genética experimental permitieron relacionar el conocimiento del sujeto al manejo de los objetos, y el conocimiento de los objetos a los fines del sujeto.

Las clasificaciones surgen del manejo de los objetos. Las clasificaciones también surgen de los valores, normas, metas y creencias de quienes manejan o construyen los objetos.

\section{LOS CONCEPTOS DEL TERMOS- TATO: LAS MÁQUINAS QUE CLASIFICAN}

¿Es posible pensar en máquinas que tengan conceptos? Claro que sí. El termómetro nos da información: codifica información. Es un agente perceptor. El termostato clasifica la temperatura en dos clases, frío y caliente. Es un agente cognitivo.

"El termómetro —escribe el lógico Devlin ${ }^{4}$ _ no tiene un concepto de lo frío y lo caliente. El termostato sí tiene un concepto de lo frío y lo caliente". Maneja las categorías, las clases, los géneros; procesa la información, la clasifica, y actúa en consecuencia: enciende, apaga, usa y ahorra energía.

Tan sugerente analogía entre los conceptos del hombre y de las máquinas plantea avanzadísimos problemas de una tecnología científica que forma parte del mundo actual. Las nuevas ciencias, las ciencias cognitivas, la bioepistemología, la robótica nos acercan a un mundo real y virtual en que somos capaces de producir una inteligencia artificial con máquinas que a su vez son capaces de construir conceptos y redes conceptuales, y de ayudarnos en las definiciones y clasificaciones de los mismos.

Lo que no podemos olvidar es que nosotros programamos a los robots, incluso cuando se reprograman a sí mismos. Usar de ellos y de las computadoras en general, facilita enormemente nuestra tarea de definir, clasificar, comprender y construir el Universo-Biblioteca, pero no nos quita la responsabilidad de pensar por nuestra propia cuenta y de asumir nuestros propios actos perceptores, cognitivos y volitivos.

\section{LAS CATEGORÍAS O LA CLASIFI- CACIÓN DE LOS FILÓSOFOS}

Los filósofos tradicionales clasifican el Ser y la Razón como fenómenos invariables o eternos. Desde Aristóteles hasta Kant — para no ir más lejos_ se establecen las diferentes clases del ser y de los predicados que se aplican a un sujeto; o las diferentes clases de conceptos fundamentales que representan el entendimiento humano. Las categorías sólo en Bacon aparecen como fenómenos experimentales, en Hegel como fenómenos históricos, y también como sociales y estructurales en Marx y Weber.

En todo caso las categorías del Ser, de la Razón, de la historia y la sociedad no se quedan en un terreno teórico de definiciones y clasificaciones conceptuales o de análisis lógicos. Reflejan, además diversas relaciones con la práctica, con las perspectivas, con los valores, intereses y organizaciones de quienes determinan cuáles son las diferentes clases del ser social y cuáles los predicados más significativos.

Las clasificaciones o categorías de "creyentes y paganos", "negros y blancos", "arios y judíos", "proletarios y burgueses", "ricos y pobres", "colonialistas y colonizados"; o las de "ciencias exactas" y "ciencias humanas"; ciencias físico-matemáticas, ciencias químicas y biológicas, etcétera, encierran profundas diferencias teóricas, ideológicas y en "las prácticas sociales". El más alto nivel filosófico nos lleva a pensar hoy que, sin lugar a dudas, toda clasificación es histórica y relativa, y toda clasificación está sujeta a la práctica de quienes la emplean.

No existe ni existirá nunca La Clasificación por antonomasia, perfecta, absoluta. El relativismo epistemológico e histórico invita a pensar qué se busca construir o qué construcción y organización buscan descubrir y hacer funcionar y echar a andar con cada clasificación los sujetos individuales y colectivos que la emplean.

\section{LA HISTORIA DE LAS CLASIFI- CACIONES Y LAS DEFINICIONES}

La historia nos ayuda a comprender el significado más profundo de las clasificaciones y las definiciones. Es un recurso indispensable contra la metafísica que ronda en nuestras conciencias. $\mathrm{La}$ historia, y no sólo el tiempo.

Las clasificaciones son como instantáneas que fijan géneros y 


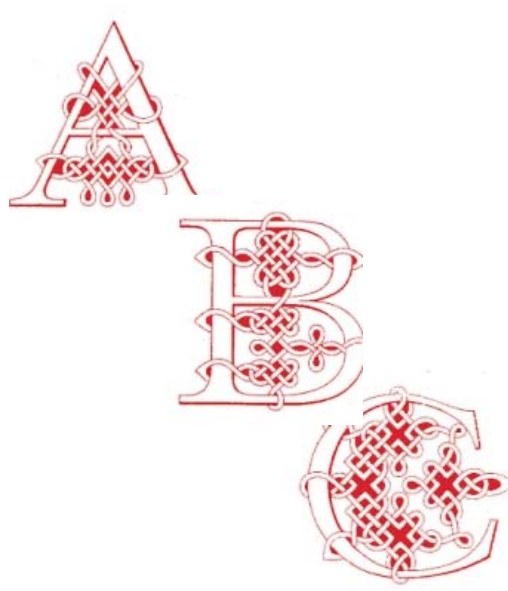

\section{"Las clasificaciones son como instantáneas que fijan géneros y diferencias, órdenes y jerarquías. Desconocer su importancia es tan grave como pensar que una de ellas constituye algo así como una verdad absoluta."}
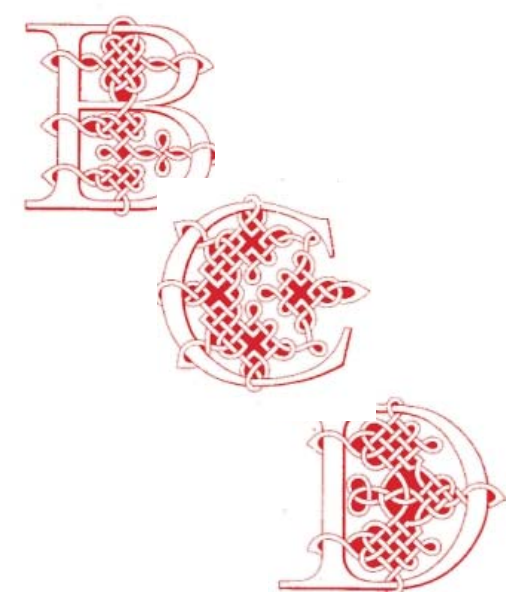

diferencias, órdenes y jerarquías. Desconocer su importancia es tan grave como pensar que una de ellas constituye algo así como una verdad absoluta.

La herencia de Aristóteles llega hasta la postmodernidad y pasa por el marxismo y el empirismo.

La definición por clasificación de algo o de alguien en un género, señalando su diferencia específica, corresponde hasta nuestros días a visiones esencialistas que creen encontrar o haber encontrado un sistema de clasificación natural con jerarquías también naturales, tras las cuales se encuentran supuestas esencias.

Cuántas polémicas inútiles se dan por esa forma de pensar o de conocer que dominó hasta fines de la Edad Media, y que entró en crisis con las relaciones y funciones de Newton, con la evolución y la genética de Darwin, y con la historia contradictoria de Marx, no se diga ya con las más recientes corrientes postmodernas y constructivistas.

Pero si hoy la clasificación no ocupa el lugar privilegiado que ocupó en el mundo clásico sigue siendo muy importante conforme se mueve uno de la física, a la química, a la biología y a las ciencias humanas. La taxonomía en biología, la tipología, la morfología y el estructuralismo en ciencias humanas, la localización en ciencias de la información y la catalogación en bibliotecología dan una idea de la enorme importancia que las clasificaciones y definiciones tienen en ciencias y humanidades. Su uso para la buena marcha de los Estados, de las empresas y las organizaciones de la sociedad civil nos indica algo sorprendente: no hay civilización sin clasificación, como tampoco hay clasificación sin historia.

La historia de las clasificaciones nos afirma en la idea de que sirven para ordenar el pensamiento y las cosas, y que varían en los distintos tiempos y culturas. Las variaciones no se deben sólo a los intereses prácticos de quienes clasifican y definen, sino a las variaciones reales de los conjuntos y los subconjuntos, de los universos y partes en que se mueven, y que se mueven.

La evolución de las estructuras y los sistemas y de los grupos, estratos, tipos, clases, genera interacciones entre dos o más subconjuntos. Esas interacciones llegan a ser más importantes que los subconjuntos de que surgieron.

A menudo, las divisiones aparecen como una especie de "clases inexactas" o de "conjuntos borrosos", pero su interacción con los clasificadores obliga a éstos a reformular sus esquemas de clasificación y a incluir nuevas categorías y subcategorías útiles para determinados propósitos, como para la clasificación de las ciencias y las humanidades en las enciclopedias, para la clasificación de las disciplinas en las universidades y para la clasificación de los libros, revistas, documentos y material electrónico en las bibliotecas.

Un hermoso estudio sobre esta larga historia de las clasificaciones es el de Mortiner J. Adler, titulado La búsqueda de la sabiduría. Una guía de aprendizaje. En él, el autor nos hace ver que las tres clasificaciones - las de las enciclopedias, las de las universidades y las de las bibliotecas - muestran cierta semejanza a lo largo de la historia. Y cómo no iban a mostrarla si todas buscan poner orden en el universo y todas tienen que ver con los descubrimientos técnicos que reorganizan el trabajo intelectual y material desde Gutenberg con su imprenta hasta Von Neumann con su computadora.

La percepción de la clasificación como historia exige no reparar sólo en los cambios sino en las duraciones, algunas largas como decía Braudel. Así, la Biblioteca del Congreso de Estados Unidos mantuvo hasta 1901 el orden que le había dado Jefferson, con la clasificación de las facultades humanas que Jefferson había tomado de Bacon, quien las dividiera en: memoria, imaginación y razón. Sólo después de más de tres siglos la Biblioteca del Congreso abandonó el legado de Bacon e inició un cambio de clasificación que "llegó a feliz término diez años después". Otra es la historia de la clasificación que aplicó a la biblioteca de su colegio Melvil Dewey en 1873 y que varias décadas más tarde se convirtió en el sistema decimal Dewey. En ese caso la clasificación no se quedó inmóvil, y no sólo se enriqueció con una nueva categoría, sino que replanteó el problema de las categorías de Bacon y transformó lo que era un orden ascendente en un orden descendente. 
Esas y otras experiencias nos revelan que ni en las bibliotecas ni en las clasificaciones hay sólo un "ámbito sereno", un tiempo "conservado", que parece "disecado" y que es primero "mágico" y después técnico.

Las teorías de la biblioteca se vinculan a las de las Ciencias y las Humanidades, a lo largo de la historia de unas y otras.

Hoy, con la creciente interdisciplina entre ciencias y humanidades, con los cambios de géneros literarios y científicos, la nueva organización del conocimiento en la biblioteca exige replanteamientos muy fuertes, precisos, útiles para las divisiones $\mathrm{y}$ asociaciones del trabajo emergente que adquiere vida propia y a menudo mayor atención y esfuerzo que en el pasado.

La revolución científica iniciada a mediados del siglo XX nos obliga a replantear hoy la clasificación de las ciencias y las humanidades con una nueva teoría de la conexión entre unas disciplinas o especialidades y otras, con una nueva teoría del conocimiento que asuma el problema de los sistemas complejos en que unos elementos definen a otros y son definidos por ellos, y con una nueva teoría del aprendizaje en que métodos y contenidos, teorías y prácticas, reflexiones y experimentos interdisciplinarios nos permitan diseñar modelos, y construir conocimientos en que la verdadera clasificación —como dijo Piaget- sea "una espiral sin fin", una espiral móvil, con variaciones y constantes que deben precisarse.

La vieja división entre las Ciencias de la Naturaleza y las Ciencias de la Historia como dos tipos de conocer esencialmente distintos se vuelve obsoleta. El acercamiento de las ciencias de la materia, de las ciencias de la vida y de las ciencias humanas va más allá de las analogías, hacia isomorfismos que aclaran y precisan la unidad y diversidad del Universo, con profundas implicaciones en la biblioteca. El acercamiento de la físico-química, de la biofísica, de la bioquímica, de la zoopsicología, de la biopsicología, de la biosociología aumenta la incertidumbre de las acotaciones anteriores y de los códigos correspondientes. ¿Dónde clasificamos la cibernética? Es una ciencia de la naturaleza y también es una ciencia del hombre. ¿Dónde clasificamos la lógica cuando ésta se vincula a las matemáticas, al lenguaje, a la experimentación y a la historia, y es una ciencia tan exacta que "ni parece humana”? ¿Seguimos usando la categoría de "ciencias exactas" cuando la física teórica no es siempre una ciencia exacta? ¿Seguimos hablando de físicomatemáticas cuando las matemáticas están muy lejos de quedar adscritas a la física y se aplican cada vez más a las ciencias biológicas y humanas?

El cambio sigue. La historia está desfilando frente a nosotros. Todo se mueve. Hay categorías que hasta hace poco eran centrales y que han dejado de serlo o se han redefinido mientras otras resisten, se quedan y hasta acentúan sus rasgos más desiguales, sus distribuciones más injustas. Sabemos que lo único cierto es lo relativo de las clasificaciones. ¿Pero cuáles clasificaciones nos pueden ser de más utilidad en el momento histórico actual y para qué? Y si tenemos que hacer otras nuevas ¿cómo acercarnos a ellas? ¿Cómo construirlas? Tal vez lo mejor es pensar siempre que los libros pueden ser objeto de clasificaciones múltiples. Que siempre clasificamos con fines prácticos, de trabajo y gobierno, de información, educación y sociedad civil organizada, y que dentro de esos fines buscamos órdenes que sean racionales, funcionales, útiles, con capacidad de integrar nuevas ramas del saber y nuevos conocimientos. Un orden con varios órdenes, o varios órdenes en conflicto y coexistencia.

La historia nos enseña cómo cambian y se conservan las clasificaciones y definiciones. También cómo emergen y se construyen otras nuevas y en qué circunstancias, para qué fines.

\section{EN CIENCIAS EXPERIMENTALES SE HACEN DEFINICIONES Y CLA- SIFICACIONES QUE BUSCAN SER EXACTAS}

La búsqueda de frecuencias, de distribuciones, de tendencias de un fenómeno variable lleva a definiciones precisas de sus características y del espacio y tiempo en que ocurre. La búsqueda de correlaciones entre dos o más variables se propone esos mismos objetivos y especifica el comportamiento de las variables en distintas condiciones.

La búsqueda de definiciones y clasificaciones precisas que permitan extrapolar tendencias o calcular coeficientes de correlación parece un

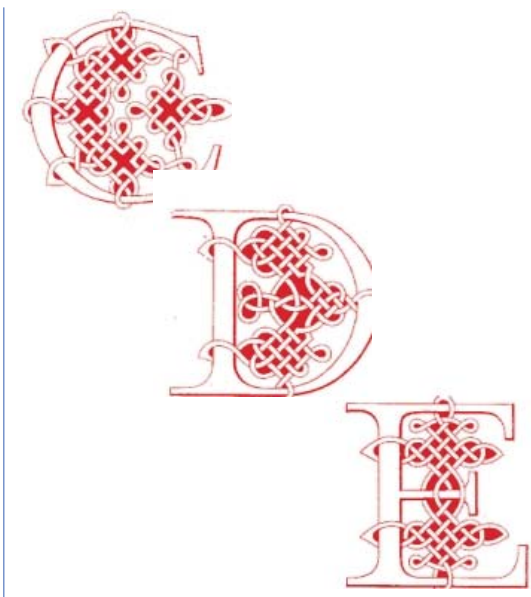

"Ni hay una sola clasificación; ni hay una sola manera de clasificar. Es más, por preciso que se sea, hay libros que con dificultad pueden ser clasificados en una categoría clara; caen en categorías de intersección, borrosas, ambiguas y no por ello menos importantes."

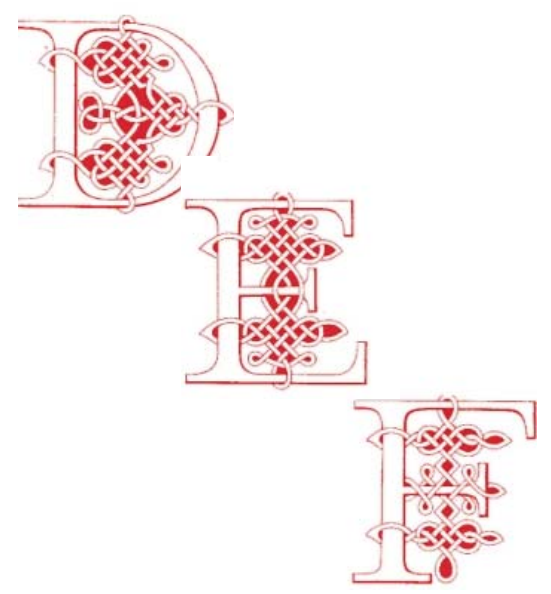


objetivo difícil de entender; pero de hecho es muy fácil, y vale la pena entenderlo para varios fines:

1. Para precisar los intervalos o límites en que se separa un grupo de otro, o una clase de otra, y

2. Para aprender a clasificar un mismo objeto según dos o más dimensiones.

En el primer caso se plantea el problema de precisar los límites o rupturas o discontinuidades de los distintos grupos en que se divide una variable y que sirven para saber con exactitud a qué grupo corresponde un objeto dado al que se quiere clasificar de acuerdo con esa variable: la clasificación estadística por "grupos de ingreso" puede ser de: 1 , menos del salario mínimo; 2, del salario mínimo y menos de \$1999.99; 3, de \$ 2,000-2999.99, etcétera. La clasificación estadística del profesional puede precisar a quienes incluye y a quienes no incluye el estrato social correspondiente para que así no haya duda: por ejemplo, quienes son y quienes no son empleados, trabajadores, empresarios. El problema de los límites precisos y los límites borrosos de los estratos o conjuntos es significativo para cualquiera que trabaja en clasificaciones; en la técnica de clasificar objetos, por ejemplo, libros. Las definiciones estadísticas ayudan a resolver ese problema incluso cuando no se trata de cuantificar los objetos clasificados.

Otro problema importante consiste en acostumbrarse a que un mismo objeto se puede clasificar en dos o más dimensiones, lo que significa que un conjunto de objetos puede caber en apartados distintos si cada dimensión se divide en dos o más clases.

Todos los libros pueden ser clasificados de acuerdo con dos o más descriptores según los objetivos de las dimensiones o variables que sirven para clasificarlos.

La conclusión es clara y no debemos olvidarla por más que nuestra tradición metafísica nos lleve a pensar en una sola clasificación como la buena. Ni hay una sola clasificación; ni hay una sola manera de clasificar. Es más, por preciso que se sea, hay libros que con dificultad pueden ser clasificados en una categoría clara; caen en categorías de intersección, borrosas, ambiguas y no por ello menos importantes. Las ciencias empíricas y experimentales nos ayudan a resolver muchos de esos problemas.
Los libros polisémicos forman parte de la vida que se crea y recrea. Afortunadamente no todos se dejan disecar; muchos nos obligan a repensar clasificaciones y codificaciones, sin preocuparnos en exceso por ciertas ambigüedades del flujo vital que encierran.

\section{RECOMENDACIONES A LOS ENCARGADOS DEL UNIVERSO}

¿Qué recomendaciones se pueden hacer a los encargados de la biblioteca en relación con las clasificaciones y definiciones y con las experiencias señaladas? Voy a enunciar algunas:

1. El bibliotecario no debe pensar en la clasificación tan sólo como un proceso por el que reparte los libros en los subconjuntos de la biblioteca, proceso cuyas características se identifican en general con la clasificación de los libros.

Es importantísimo que clasifique libros y documentos para localizar la información que en un momento dado requieren los usuarios. Pero si se interesa en el orden de su biblioteca necesita pensar en la clasificación como una forma de ordenar los conceptos en relaciones alfabéticas y cronológicas y en otras de género o especie, de calidad o jerarquía, de calidad o de tamaño. En este terreno, el bibliotecario puede incluso dar un paso más al vincular la clasificación de su biblioteca a la del saber humano y sus formas de dividirse, definirse, clasificarse en la investigación, la educación, la sociedad y el Estado. El gran bibliotecario sabrá clasificar para distinguir unos libros y conocimientos de otros y también para relacionar unos libros y conocimientos con otros: las humanidades, las ciencias, las ingenierías y las tecnologías.

2. El acervo de libros y documentos se hace en función de ideologías e intereses y los archivos y bibliotecas generales contienen documentos y libros de las más opuestas corrientes y escuelas. Como universo del saber y el polemizar todos deben ser preservados.

3. La clasificación de los documentos y libros y la definición de cada categoría plantea distintos tipos de problemas ideológicos y técnicos. En- tre los ideológicos destacan las distintas formas de concebir y dividir el mundo con categorías tabú que se ocultan o prohíben y categorías oficiales $u$ ortodoxas que obligan a clasificar de una manera determinada. Entre los problemas técnicos se encuentran las fronteras o límites borrosos entre una categoría y otra, así como las intersecciones de dos o más categorías. Entre los problemas críticos se encuentran las categorías significativas y los autores importantes del pensar alternativo.

4. La clasificación y la definición no sólo enfrentan problemas ideológicos y técnicos sino los que caracterizan a la evolución histórica y social. Hay categorías emergentes y otras en proceso de extinción; rasgos que fueron característicos de un fenómeno y que dejan de serlo. Aferrarse a las categorías, clasificaciones y definiciones de antes sin registrar los cambios de ahora puede ser inconveniente para la organización del conocimiento, para el almacenamiento y para la consulta de la información. Lo es desde luego para un proceso educativo en que el estudioso requiere acercarse a los problemas desde las categorías y definiciones vivas, actuales.

5. El almacenamiento y la recuperación automatizada de datos permite resolver muchos de los problemas señalados. La clasificación de un mismo documento o libro en distintas categorías es tan fácil hoy como el incluir una gran cantidad de criterios y dimensiones para la clasificación.

Ni una sola clasificación, ni una sola definición, ni un solo código puede imponerse sobre los demás. Buscar y aplicar los más significativos para la investigación, la docencia y la difusión de la cultura es una tarea primordial para poner orden o encontrar las distintas formas de ordenar datos y conocimientos.

El problema principal sigue siendo ideológico con categorías tabú que suelen no incluirse y categorías engañosas o desestructuradas que se incluyen.

Sólo los bibliotecarios filósofos y los bibliotecarios críticos que busquen un pleno respeto al pluralismo 
ideológico, cultural, religioso, político lograrán un día imponer todas las clasificaciones significativas.

6. Un problema no menos importante es el del ruido en la información, el del exceso de información que recuperan las computadoras si no se define con claridad el perfil de interés del lector. La precisión del espacio conceptual por el lector y las posibilidades de ampliarlo y reducirlo de acuerdo con sus deseos de contextualizar el conocimiento se facilitarán cuando las clasificaciones y codificaciones de las bibliotecas se formulen y reformulen de acuerdo con la evolución del trabajo intelectual disciplinario e interdisciplinario; universal, regional y nacional; general y especializado.

Archivos y bibliotecas pueden ajustarse a la dinámica de la historia si usan las nuevas tecnologías y ciencias de la información, y se combinan con reflexiones prácticas y de sentido común.

A la elaboración de un buen perfil de interés por el usuario se puede añadir la codificación de libros y documentos que se recomiendan para una lectura pedagógica a quienes desean iniciarse en el estudio de un problema, o fenómeno determinado, de una disciplina o de una especialidad interdisciplinaria.

Organizar el trabajo de libros y textos que se recomiendan con sentido pedagógico, es un trabajo descuidado por la administración de las universidades y de las bibliotecas. Requiere una alternativa muy especial en las actuales condiciones de la información y el conocimiento. Es importantísimo para la política de adquisición de una biblioteca en que se escogen "los mejores", los más "significativos" para la cultura general y las especialidades, a partir de listas de recomendación de lecturas, o de notas y artículos que destacan sus virtudes.

Escoger las fuentes de selección de libros plantea problemas tan importantes como la selección de los libros. Es necesario asumirlos para acercarse constantemente a la mejor selección en un campo y momento dados.

7. El bibliotecario, el autor de un Thesaurus, y el maestro que diseña un plan de estudios, así como el filósofo y el investigador que se ocupan de definir y clasificar las ciencias y las humanidades descubren tarde o temprano que no existe la definición perfecta ni la clasificación perfecta. Problematizar su tarea, cuestionar sus ideas será siempre muy importante si ellos mismos hacen tres cosas:

1) Buscar una solución práctica y para eso, 2) Informarse sobre las soluciones que se han dado a los problemas que se les plantean y 3) Pensar en problemas concretos y en soluciones concretas sin quedarse en vagas dificultades o complejidades inhibitorias, que hacen pensar en una especie de saber que uno nunca logrará.

8. Si la Biblioteca es el Universo, el conocimiento relativo que tenemos del Universo será un conocimiento también relativo de la Biblioteca. El problema es que ese conocimiento sea lo más riguroso, exacto, claro, práctico y útil que sea posible.

Y si en la Biblioteca hay un orden, es importante no convertir ese orden en un dogma sino dejar que en él quepan las más distintas corrientes del pensamiento que lo definen, dividen, clasifican y mueven, y que a través de él lleguen quienes quieren conocer el universo o una parte del Universo y que encuentren lo que sabemos o creemos saber del todo y lo que sabemos.

En ese sentido es muy importante - como guía del Universo y de la biblioteca- enseñar al lector que entra por vez primera a manejar el catálogo de la biblioteca y de las redes que la conectan con las bibliotecas del mundo. También puede ser importante enseñarle algunos libros muy hermosos como La Divina Comedia ilustrada por Gustavo Doré, o colecciones en que puede leer grandes obras como Nuestros Clásicos o libros que acaban de llegar y que le permiten actualizar o ampliar sus conocimientos.

En la Universidad el estudiante, en la Enciclopedia el usuario, en la biblioteca el lector constituyen la parte más importante de ese universo cuyos objetos define, clasifica y recupera el profesor, el enciclopedista y el bibliotecario.

Los textos "no existen en los libreros..." Como ha escrito Terry Eagleton "corresponden a procesos de significación materializados en la práctica de la lectura". Dar existencia a los libros es ayudar — con la magia del orden, de las clasificaciones, definiciones, codificaciones, elecciones-a sacarlos de sus libreros y facilitar la práctica de la lectura entre los usuarios del Universo. Esa modesta tarea fue la de un Borges, gran poeta y constructor de nuestro tiempo, y es la de todos los buenos documentalistas y bibliotecarios. La parte humana del acercamiento al Universo resulta por eso inolvidable para cualquier lector, como para mí lo es la figura de don Juan B. Iguíniz que me llevaba a ver los incunables de la Biblioteca Nacional, o la de don Antonio Pompa y Pompa que fruncía el seño por los retrasos en la devolución de los libros y, tras regañarme, me prestaba con entusiasmo otros nuevos, o la de Susana Uribe, que en el Colegio de México mostraba más amor por los libros que por los lectores, a quienes, sin embargo, guiaba con una gran frialdad y eficacia. A todos ellos les guardo un profundo agradecimiento por haberme hecho vivir en sus universos, y con mi homenaje a su memoria termino esto. 